\title{
Numerical simulation of long-path spherical wave propagation in three-dimensional random media
}

\author{
Juan Antonio Rubio \\ Aniceto Belmonte \\ Adolfo Comerón, MEMBER SPIE \\ Universidad Politécnica de Cataluña \\ Department of Signal Theory \\ and Communications \\ Campus Nord UPC-Edif. D3 \\ Jordi Girona 1-3 \\ 08034 Barcelona \\ Spain \\ E-mail: rubio@tsc.upc.es
}

\begin{abstract}
A new method to overcome some limitations in the simulation of the propagation of waves originating from a point source through a very long path in a turbulent medium is presented. Existing propagation simulation algorithms suffer from either windowing or lack of resolution when applied to long paths. If Cartesian coordinates are used, the limited size of the numerical mesh eventually leads to windowing errors. Casting the classical split-step Fourier algorithm in a spherically diverging coordinate system allows one to get around this problem. In this way an angular mesh matching the source and the propagation algorithm to the problem geometry is used. But for long-path propagation, this spherical divergent mesh causes a loss of resolution that can become a serious problem in the evaluation of the field statistical moments. The method discussed in this paper overcomes both the windowing effect associated with Cartesian coordinates and the loss of resolution accompanying spherical coordinates by using a spherical-coordinate algorithm and performing repeated interpolations of the numerically propagated field before the mesh grows too large to sample the field accurately. Each time an interpolation is done, the angular window is decreased to maintain the matrix size. (c) 1999 Society of Photo-Optical Instrumentation Engineers. [S0091-3286(99)00309-8]
\end{abstract}

Subject terms: atmospheric propagation; split-step algorithm.

Paper 980411 received Oct. 28, 1998; revised manuscript received Mar. 2, 1999; accepted for publication Mar. 10, 1999.

\section{Introduction}

The method of smooth perturbations or Rytov's theory correctly describes the effects of atmospheric turbulence on the propagation of electromagnetic waves when the fluctuations of the logarithmic amplitude (log amplitude) are small. ${ }^{1}$ These fluctuations can be considered small when the propagation paths are sufficiently short or the turbulence is very weak. Asymptotic theories that can predict the effects of very strong atmospheric turbulence have also been developed and checked. ${ }^{2}$ Between these two limiting cases lies the so-called strong-focusing peak region, for which a reliable analytical theory does not exist. In this turbulence range, numerical simulation is the best tool for predicting how the index-of-refraction fluctuations will affect the wave propagation.

The first simulations of the propagation of spherical waves in three-dimensional random media used the central part of a Gaussian beam source in a Cartesian grid for simulating the point source. ${ }^{3}$ Applying the Fourier splitstep algorithm in such a Cartesian coordinate system limited its use to short paths, due to the windowing effect on the field caused by the fixed numerical mesh. Casting the classical split-step Fourier algorithm in a spherically diverging coordinate system can avoid the windowing problem, but when long-path propagation is simulated, errors in the evaluation of the field statistical moments arise because of the lack of resolution in the sampled field. To illustrate the effect of insufficient resolution in the evaluation of field moments, let us consider the following example. Assume a 4-km path along which a uniform, low enough turbulence strength exists, producing a Rytov log-amplitude variance $\sigma_{\chi}^{2}=0.05$. Define $R_{f}=\left(\lambda L_{z t}\right)^{1 / 2}, \lambda$ being the radiation wavelength, which we take as $1 \mu \mathrm{m}$, and $L_{z t}$ the path length. Let us apply the classical split-step Fourier propagation algorithm with 20 propagation steps whilst keeping a given number of sample points. If the spatial sampling period $\Delta x$ is taken as $\Delta x=R_{f} / 50$, the error in the computed log-amplitude variance is around $10 \%$; if $\Delta x$ is taken instead as $\Delta x=R_{f} / 2$, the computation error jumps to $50 \%$.

In this paper we propose a new algorithm to overcome the windowing and loss-of-resolution problems, making good use of the spherical split-step Fourier algorithm and performing repeated interpolations of the numerically propagated field before the mesh grows too large to accurately sample the field. When an interpolation is done, the angular window is reduced to maintain the size of the matrices. In Sec. 2 we describe the simulation algorithm proposed for propagating spherical waves in three-dimensional random media. In Sec. 3 some simulation results are compared with the theoretical results available in the weak turbulence range, and some propagation experiments found in the literature are simulated to study the performance of the simulation tool. Section 4 presents the conclusions. 


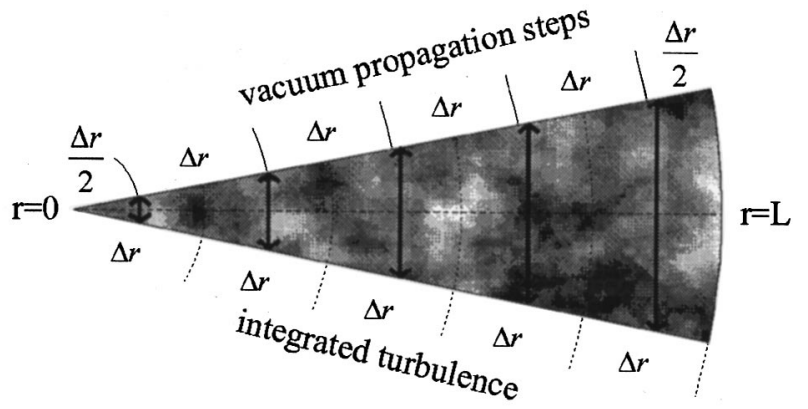

Fig. 1 Outline of the algorithm for a spherical divergent coordinate system. First the symmetry is introduced with a vacuum propagation from $r=0$ to $r=\Delta r / 2$. Next the phase change due to the integrated turbulence between $r=0$ and $r=\Delta r$ is applied. The algorithm consecutively applies vacuum propagation steps over a distance $\Delta r$ and phase changes in accordance with screens of thickness $\Delta r$, finishing with a vacuum propagation step over a distance $\Delta r / 2$.

\section{Description of the Simulation Algorithm}

\subsection{Treatment of the Propagation Equation}

The numerical simulation of wave propagation in random media starts with the parabolic or Fresnel approximation of Maxwell's wave equation. ${ }^{4}$ Considering a spherically divergent coordinate system $(r, \theta, \phi)$ defined by $x=r \theta, y$ $=r \phi$, where $r$ is the propagation distance and $\theta, \phi \ll 1$, the parabolic wave equation can be written as

$\frac{1}{r^{2}} \nabla_{T}^{2} E-2 j k \frac{\partial E}{\partial r}+2 k^{2} n_{1} E=0$,

where $k=2 \pi / \lambda$ is the wave number, $n_{1}$ is the deviation of the index of refraction from its mean value $\bar{n}$, and $\nabla_{T}^{2}$ $=\partial^{2} / \partial \theta^{2}+\partial^{2} / \partial \phi^{2}$.

Solving (1) allows us to propagate an initial field from the spherical cap $r$ to an arbitrary cap $r+\Delta r$ :

$$
\begin{aligned}
E(r+\Delta r, \theta, \phi)= & \exp \left\{-j\left[\frac{\nabla_{T}^{2}}{2 k}\left(\frac{1}{r}-\frac{1}{r+\Delta r}\right)+\Delta S_{n}\right]\right\} \\
& \times E(r, \theta, \phi),
\end{aligned}
$$

where $\Delta S_{n}=k \int_{r}^{r+\Delta r} n_{1} \mathrm{~d} r^{\prime}$ can be interpreted as the phase change introduced by the index-of-refraction inhomogeneity associated with the turbulence. The use of the symmetrized split-step operator ${ }^{4}$ allows splitting the algorithm with second-order accuracy. The propagation of the field over a distance $\Delta r$ is decomposed into a vacuum propagation over a distance $\Delta r / 2$, an upgrading of the phase in accordance with the random medium changes, and a second vacuum propagation of the resulting field over a distance $\Delta r / 2$. After the first vacuum propagation introduced by the split-step operator, the half steps of propagation can be combined into single propagation steps, resulting in a symmetrical algorithm. A random-screen algorithm implements the turbulence-induced random phase shift.

In Fig. 1, an outline of the spherical divergent coordinate system algorithm is shown. First, as commented before, the symmetry is introduced with a vacuum propagation from $r=0$ to $r=\Delta r / 2$. Next the phase change due to the integrated turbulence between $r=0$ and $r=\Delta r$ is applied. The algorithm consecutively applies vacuum propagation steps over a distance $\Delta r$ and phase changes in accordance with screens of thickness $\Delta r$, finishing with a vacuum propagation step over a distance $\Delta r / 2$.

The homogeneous equation $\left(n_{1}=0\right)$

$\frac{\partial E}{\partial r}=-j \frac{\nabla_{T}^{2}}{2 k r^{2}} E$

is solved in the Fourier-transform domain with the fast Fourier transform (FFT) algorithm. If $\widetilde{E}$ is the Fourier transform of the field, then

$\frac{\partial \widetilde{E}}{\partial r}=\frac{j}{2 k r^{2}}\left(K_{\theta}^{2}+K_{\phi}^{2}\right) \widetilde{E}$,

where $K=\left(K_{\theta}, K_{\phi}\right)$ is the transformed angular variable. Finally, the vacuum propagation is given by

$$
\begin{aligned}
\widetilde{E}\left(r+\Delta r, K_{\theta}, K_{\phi}\right)= & \exp \left[-\frac{j}{2 k}\left(K_{\theta}^{2}+K_{\phi}^{2}\right)\right. \\
& \left.\times\left(\frac{1}{r+\Delta r}-\frac{1}{r}\right)\right] \widetilde{E}\left(r, K_{\theta}, K_{\phi}\right),
\end{aligned}
$$

which will be used for propagating the field from each phase screen to the next.

The field can be considered as a band-limited random process, and we can use sampling theory to choose the appropriate grid spacing in order to get a discrete representation of the field without loss of information. ${ }^{5}$ If $\Delta \theta$ $=L_{\theta} /(N-1), \Delta \phi=L_{\phi} /(N-1)$ are the angular resolutions used to sample the field, $N \times N$ is the number of points in the numerical mesh, and $L_{\theta} \times L_{\phi}$ is the simulation window angular dimension, then the Fourier transform of the field computed through the fast Fourier transform (FFT) will show the angular frequencies $K_{m \theta}=\left(2 \pi / L_{\theta}\right) m, K_{n \phi}$ $=\left(2 \pi / L_{\phi}\right) n(m, n=1, \ldots, N)$. The discrete Fourier transform imposes on the fields a periodicity that must be controlled in order to avoid aliasing.

The field in $r+\Delta r$ is obtained by applying Eq. (5) to the sampled field and performing the inverse Fourier transform with a FFT algorithm:

$$
\begin{aligned}
\widetilde{E}\left(r+\Delta r, K_{m \theta}, K_{n \phi}\right)= & \exp \left[-\frac{j}{2 k}\left(K_{m \theta}^{2}+K_{n \phi}^{2}\right)\right. \\
& \left.\times\left(\frac{1}{r+\Delta r}-\frac{1}{r}\right)\right] \widetilde{E}\left(r, K_{m \theta}, K_{n \phi}\right),
\end{aligned}
$$

$E(r+\Delta r, p \theta, q \phi)=\operatorname{IFFT}\left\{\widetilde{E}\left(r+\Delta r, K_{m \theta}, K_{n \phi}\right)\right\}$. 


\subsection{Treatment of the Atmospheric Turbulence}

The propagation of a wave through a continuous random medium is the limiting case of propagation through a discrete series of random phase screens separated by free space. To properly approximate the continuous-medium propagation using a finite number of screens, the phase shift due to each screen must be small. Decomposing the medium into several independent weak phase screens is equivalent to the Markov approximation, ${ }^{1}$ usually used in the analytical approaches to problems of this kind.

The passage of a wave $E(r, \theta, \phi)$ through a thin screen introducing a phase change $\Delta S_{n}$ in $r=r_{c}$ can be expressed as

$E\left(r_{c}^{+}, \theta, \phi\right)=E\left(r_{c}^{-}, \theta, \phi\right) \exp \left(-j \Delta S_{n}\right)$.

The random medium defined by the thin screen between $r=r_{c}-\Delta r / 2$ and $r=r_{c}+\Delta r / 2$ can be described using the spatial spectrum of the index-of-refraction fluctuations, $\Phi_{n}\left(r, K_{\theta}, K_{\phi}\right)$. The phase fluctuations are given by

$\Delta S_{n}\left(r_{c}, \theta, \phi\right)=k \int_{r_{c}-\Delta r / 2}^{r_{c}+\Delta r / 2} n_{1}(r, \theta, \phi) \mathrm{d} r$.

The phase fluctuation spectrum $\Phi_{\Delta S_{n}}$ is related to the power spectrum of the index of refraction fluctuations, $\Phi_{n}$, through the expression ${ }^{5}$

$\Phi_{\Delta S_{n}}\left(K_{\theta}, K_{\phi}\right)=2 \pi k^{2} \int_{r_{c}-\Delta r}^{r_{c}+\Delta r} z^{-2} \Phi_{n}\left(K_{z}=0, \frac{K_{\theta}}{r}, \frac{K_{\phi}}{r}\right) \mathrm{d} z$.

When $\Delta r \ll r_{c}$ and $\Delta r \gg L_{0}\left(L_{0}\right.$ being the outer scale of the turbulence), the expression (10) can be approximated by

$\Phi_{\Delta S_{n}}\left(K_{\theta}, K_{\phi}\right)=\frac{2 \pi k^{2} \Delta r \Phi_{n}\left(K_{r}=0, K_{\theta} / r_{c}, K_{\phi} / r_{c}\right)}{r_{c}^{2}}$.

The assumption that $\Delta r \gg L_{0}$ is equivalent to considering that there is no correlation between the turbulence in two successive phase screens (Markov approximation) and the correlation function of the index-of-refraction fluctuations has an impulsive dependence (Dirac's delta) on the propagation direction $r{ }^{6,7}$

The generation of phase screens, considering the statistics defined by Eq. (11), is performed using Monte Carlo techniques. ${ }^{8}$ After the generation of pairs of sets of pseudorandom numbers $A\left(K_{\theta}, K_{\phi}\right)+j B\left(K_{\theta}, K_{\phi}\right)$ in the frequency domain, with Gaussian distribution functions and plane power spectra (Gaussian white noise), they are filtered with the spectrum $\Phi_{\Delta S_{n}}$ of the phase fluctuations:

$\left[A\left(K_{\theta}, K_{\phi}\right)+j B\left(K_{\theta}, K_{\phi}\right)\right]\left[\Phi_{\Delta S_{n}}\left(K_{\theta}, K_{\phi}\right)\right]^{1 / 2}$.

Performing the inverse Fourier transform, two phase screens are obtained. The numbers of their numerical meshes are both autocorrelated with the desired statistics. Given the relationship (10) between the spectrum of the phase fluctuations and the spectrum of the index-ofrefraction fluctuations, the final expression for the phase screen will be

$$
\begin{aligned}
\Delta S_{n}\left(r_{c}, \theta, \phi\right)= & \left(2 \pi k^{2} \Delta r\right)^{1 / 2} \operatorname{IFFT}\left\{\left[A\left(K_{\theta}, K_{\phi}\right)\right.\right. \\
& \left.\left.+j B\left(K_{\theta}, K_{\phi}\right)\right]\left[\frac{\Phi_{n}\left(K_{\theta} / r_{c}, K_{\phi} / r_{c}\right)}{r_{c}^{2}}\right]^{1 / 2}\right\} .
\end{aligned}
$$

Although more realistic formulations for the index-ofrefraction fluctuation spectrum have been described, ${ }^{9}$ a von Kármán spectrum, given by ${ }^{10}$

$\Phi_{n}(K)=0.033 C_{n}^{2}\left(K^{2}+K_{0}^{2}\right)^{-11 / 6} \exp \left(-K^{2} / K_{m}^{2}\right)$,

has been used in the simulations for computational convenience. Moreover, as shown later, the simulation results obtained with this spectrum and the published experimental results are in fairly good agreement. In Eq. (14) $C_{n}^{2}$ is the structure constant of the index of refraction, $K_{0}=2 \pi / L_{0}$, $K_{m}=5.92 / l_{0}$, and $L_{0}$ and $l_{0}$ are the outer and inner scales of the turbulence, respectively.

\subsection{Source Definition}

A spherically divergent coordinate system allows us to efficiently approximate the spherical wave in a finite numerical mesh. We use a super-Gaussian beam to confine the angular fields. ${ }^{11}$ In a spherical coordinate system a windowed spherical wave can be represented by a quasiuniform intensity distribution given by

$E(r, \theta, \phi)=\exp \left[-\frac{1}{2}\left(\frac{\theta^{2}+\phi^{2}}{W^{2}}\right)^{8}\right]$,

which is equivalent to considering an uniformly illuminated aperture with angular diameter $2 W$, with an apodization effect at its edges for narrowing the spectrum and reducing the aliasing.

\subsection{Simulation of Long-Path Spherical Wave Propagation}

The simulation of the propagation of waves originating from a point source using spherical divergent coordinate systems avoids the characteristic windowing errors of simulations in Cartesian coordinates. Using an angular mesh adapts the source and the propagation algorithm to the problem geometry. However, when long-path propagation is simulated, this angular mesh causes a continual loss of spatial resolution that may produce severe errors in the estimated statistical moments of the propagated fields.

In this paper we propose a new simulation algorithm for circumventing this loss of resolution. The algorithm uses the spherical-coordinate system discussed before, but it performs repeated interpolations of the numerically propagated field before the mesh grows too large to sample the field accurately. Each time an interpolation is done, the angular window is decreased to maintain the size of the matrices. 


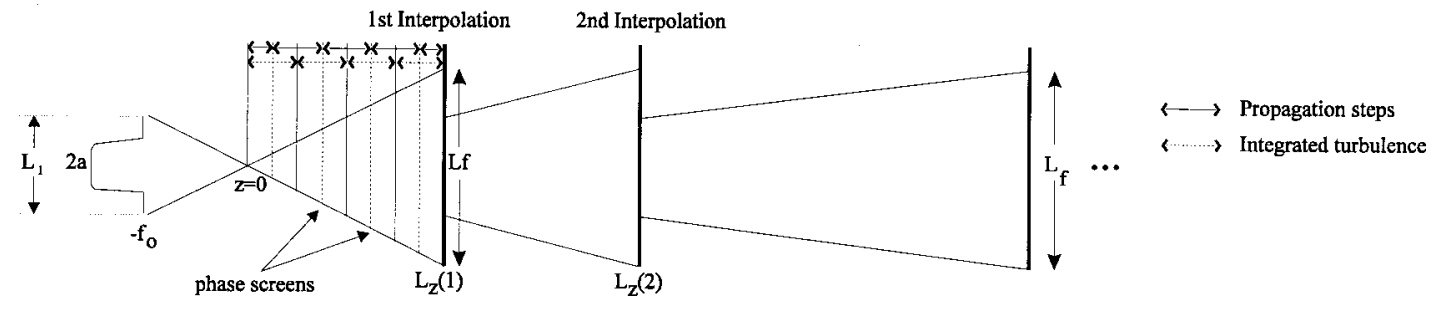

Fig. 2 Outline of the simulation algorithm for long-path spherical wave propagation.

This reduces the angular window, yet allows a sufficient size to work with appropriate Fresnel numbers to simulate the propagation until the next interpolation is performed. In this way the algorithm maintains the desired spatial resolution at the receiver plane. The angular-resolution increase is performed following the outline in Fig. 2.

The algorithm starts with the definition of the superGaussian beam at $z=-f_{0}$ to skip the point-source singularity at the coordinate origin. It is necessary to consider an appropriate relationship between the beam width $a$ at $1 / \sqrt{e}$ and the mesh width $L_{1}$. Their ratio must be small enough to prevent spurious field entering through the edges, yet large enough to avoid aliasing in the transformed domain. Usually $a / L_{1}=1 / 3$ yields good results. The desired spatial resolution $(\mathrm{d} x)$ at the receiver plane $\left(z=L_{z t}\right)$ for a fixed number of pixels in the numerical mesh $(N \times N)$ will set the spatial width of the final grid $\left(L_{f}\right)$. This width will in turn determine the condition for performing an interpolation when it is reached by the spherical-coordinate propagation algorithm (see Fig. 2). The initial width $a$ of the super-Gaussian beam must be chosen so that the beam is sufficiently sampled with $N \times N$ points and that the Fresnel number $N_{f}=a^{2} / \lambda\left[f_{0}+L_{z}(1)\right]$ corresponding to the propagation between $z=-f_{0}$ and the first interpolation plane $\left[L_{z}(1)\right]$ is large enough. A Fresnel number $N_{f}=10$ yields rms errors of the vacuum-propagated mean intensity less than $10^{-3}$.

The total number of propagation steps must be greater than 20. This will guarantee variances of the intensity fluctuations for every step less than the $10 \%$ of the total one. ${ }^{6}$ This condition can be expressed as

$\sigma_{1}^{2}(\Delta z)<0.1 \sigma_{1}^{2}\left(L_{z t}\right)$,

where $\Delta z$ is the step length, $L_{z t}$ is the total propagation distance, and $\sigma_{1}^{2}$ is the variance of the intensity fluctuations computed following Rytov's theory.

Between interpolations the spherical algorithm sketched before is applied, using the symmetrical split-step operator. To do this it is necessary to start the simulation with a half vacuum-propagation step. Next, iterative phase changes (dotted arrows in Fig. 2) computed from Eq. (8) and vacuum propagations (solid arrows in Fig. 2) are applied. Before interpolations are done, a new half vacuumpropagation step is performed.

Each time the numerical mesh grows to the spatial width limit $L_{f}$, the angular resolution is doubled by means of interpolations of the propagated field using the FFT algorithm. The interpolations are performed by first doing a zero padding in the transformed domain and then carrying out an inverse Fourier transform. ${ }^{12}$ Then the angular window is reduced to half its width to maintain the size of the matrices.

\section{Comparison with Theoretical and Experimental Results}

To evaluate the validity of the simulation, its results have been compared with available theoretical predictions. Rytov's theory allows us to assure good simulator behavior in the weak turbulence range.

Simulations in the weak turbulence range have been performed for $17.5-$ and $35-\mathrm{km}$ paths assuming a wavelength of $830 \mathrm{~nm}$ and a uniform $C_{n}^{2}$, yielding a theoretical $\log$ amplitude variance $\sigma_{\chi}^{2} \approx 3 \times 10^{-4}$ in both cases. For the $17.5-\mathrm{km}$ path we took $C_{n}^{2}=10^{-18} \mathrm{~m}^{-2 / 3}$, a final mesh width of $6 \mathrm{~m}$, inner and outer scales of $9.4 \mathrm{~cm}$ and $6 \mathrm{~m}$, respectively, and three interpolations. For the $35-\mathrm{km}$ path we took $C_{n}^{2}=2 \times 10^{-19} \mathrm{~m}^{-2 / 3}$, the same final mesh width and turbulence scales as for the previous case, and three or four interpolations. These $C_{n}^{2}$ values are admittedly veryalmost unrealistically-low, but they are only used because they yield low log-amplitude variances that can be compared with analytically computed figures. The variances estimated from the simulations were compared with the theoretical ones computed from the value at the origin ( $\rho$ $=0$ ) of the spatial covariance function of the log-amplitude fluctuations, given by

$C_{\chi}(\rho)=2 \pi \int_{0}^{\infty} \mathrm{d} K J_{0}(K, \rho) \Phi_{\chi}(K) K$,

where $\Phi_{\chi}$ is the power spectral density of the $\log$ amplitude fluctuations expressed as

$\Phi_{\chi}(K)=2 \pi k^{2} \int_{0}^{L_{z t}} \mathrm{~d} z \Phi_{n}\left(\frac{K}{\gamma}\right) \frac{1}{\gamma^{2}} \sin ^{2}\left[\frac{\left(L_{z t}-z\right) K^{2}}{2 k \gamma}\right]$

with $\gamma=z / L_{z t}$ and $\Phi_{n}$ the spectrum of the index-ofrefraction fluctuations. We have considered a von Kármán index-of-refraction spectrum to take into account the inner and outer scales of turbulence in the calculation of the theoretical variances.

Errors in the log-amplitude variance estimated from the simulations decrease with increasing number of propagation steps. In Fig. 3 the errors for different numbers of propagation steps can be seen. The simulations were performed using $128 \times 128$-point grids. The error bars in the 


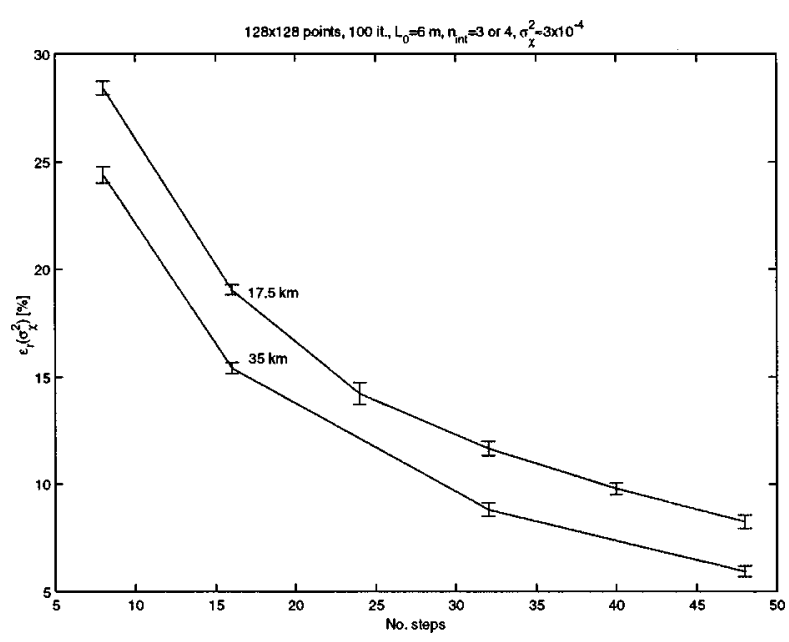

Fig. 3 Errors of the simulated log-amplitude variances versus the number of steps and the propagation distance.

error curves correspond to the standard deviation obtained from several simulations. For a $128 \times 128$-point grid and 48 propagation steps we obtained errors in the log-amplitude variances of about $8 \%$. The errors fall when the number of grid points increases, diminishing to $6.25 \%$ for $256 \times 256$ points and to $5.6 \%$ for $512 \times 512$ points. For a longer propagation path (35 km with three or four interpolations) the variance errors decrease to less than $6 \%$ for a 128 $\times 128$-point grid and 48 propagation steps.

In Fig. 4 we present a typical estimate of the normalized spatial covariance function of the log-amplitude fluctuations obtained with the simulator for a homogeneous path within a weak turbulence range. The path was $17.5 \mathrm{~km}$ long, and we used a $128 \times 128$-point grid. The curve in the figure corresponds to the theoretical covariance function computed from Eqs. (17) and (18). Good agreement between the simulated points $(+)$ and the theoretical covariance function (continuous line) is found. The error in the correlation length defined as

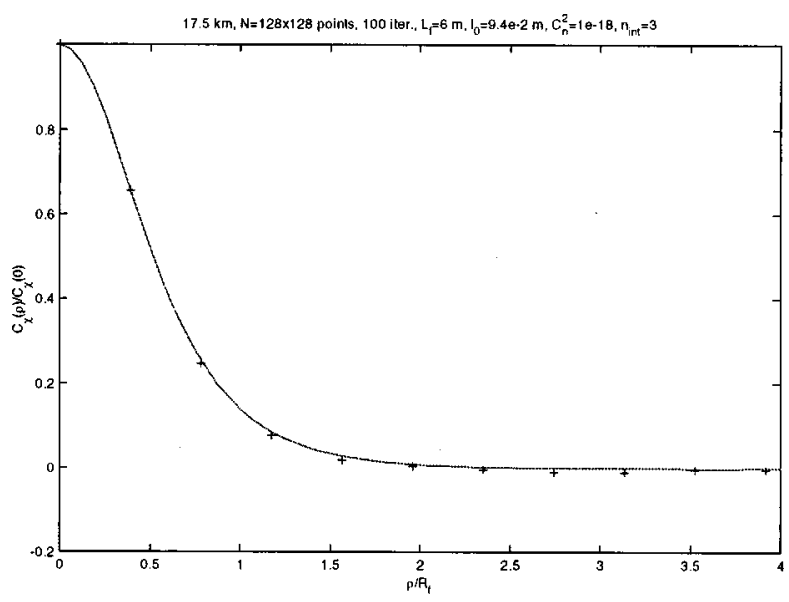

Fig. 4 Theoretical (continuous line) and simulated (crosses) normalized spatial covariance functions of the log-amplitude fluctuations. Log-amplitude variance error $=7.9 \%$, correlation-length error $=1.7 \%$.

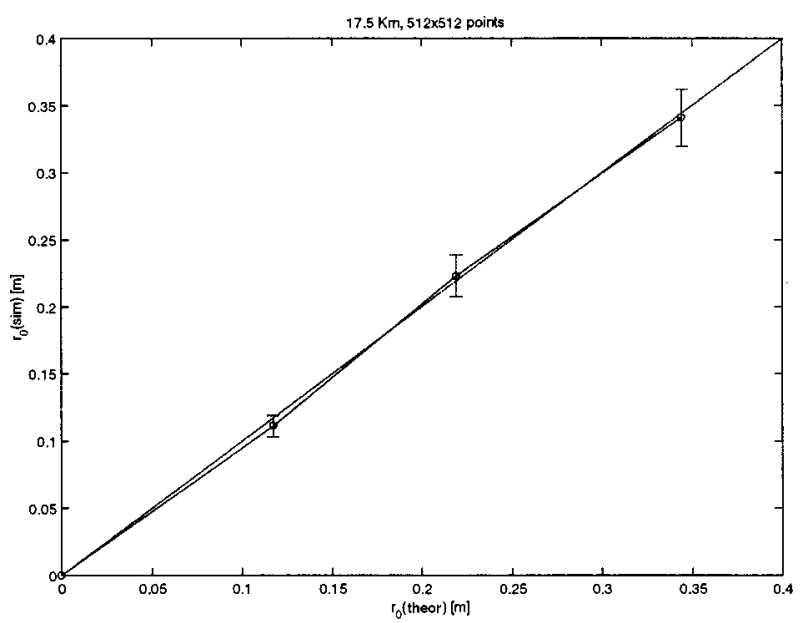

Fig. 5 Simulated versus theoretical coherence diameters. Error bars show the uncertainty due to the lack of spatial resolution.

$\rho_{c}=\frac{\int_{0}^{\infty} C_{\chi}(\rho) \mathrm{d} \rho}{C_{\chi}(0)}$

is less than $2 \%$ for a $128 \times 128$-point grid simulation, 100 iterations, and 48 steps. For larger numbers of points in the matrices the errors amount to less than $3 \%$ for a 256 $\times 256$-point grid and to approximately $4.5 \%$ for a 512 $\times 512$-point grid.

The estimation of the coherence (Fried's) diameter $\left(r_{0}\right)$ requires a more critical selection of the propagation parameters in the simulation, since it is necessary to have enough resolution to resolve this length adequately and to perform a good averaging of $r_{0}$. The suitable parameter settings can be summarized in the following conditions: $r_{0}>4 L_{0} /(N$ $-1)$ and $L_{0}>30 r_{0}$, which, for a given number $N$ of points of the numerical grid, will fix the mesh width or the maximum outer scale $L_{0}$ of the simulation.

The coherence diameters estimated from the simulations are compared with the theoretical ones, computed from the mutual coherence function $M(\rho)$ as twice the value of $\rho$ that makes $M(\rho)=1 / e$. The mutual coherence function can be expressed as

$$
\begin{aligned}
M(\rho) & =\exp \left[-\frac{1}{2} D_{w}(\rho)\right] \\
& =\exp \left\{-\left[C_{\chi}(0)+C_{\phi}(0)-C_{\chi}(\rho)-C_{\phi}(\rho)\right]\right\},
\end{aligned}
$$

where $D_{w}(\rho)$ is the wave structure function and $C_{\chi}(\rho)$ and $C_{\phi}(\rho)$ are the spatial covariance functions of the $\log$ amplitude and phase fluctuations, respectively.

In Fig. 5 we show simulated versus theoretical coherence diameters. The simulations were performed with a $512 \times 512$-point numerical mesh and a propagation distance of $17.5 \mathrm{~km}$. The error bars show the uncertainty due to the lack of spatial resolution.

We have also simulated some controlled experiments described in the literature ${ }^{13,14}$ in order to test the simulator's behavior in the saturation range. Specifically, in this paper we show some results from the simulation of Consortini et al.'s experiment described in Ref. 13. 


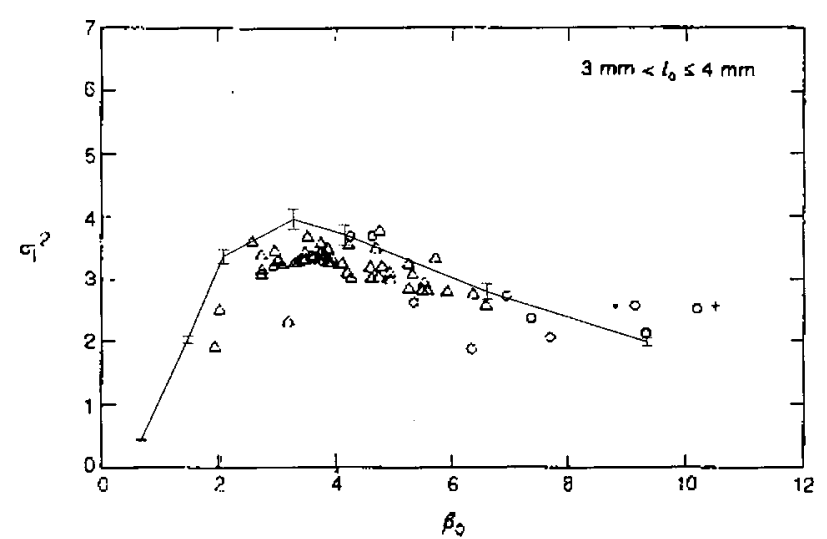

Fig. 6 Variances of the irradiance fluctuations simulated with a 3.9-mm inner scale versus $\beta_{0}$. Simulated results (points connected by lines) are shown superimposed on experimental measurements by Consortini et al. ${ }^{13}$ (triangles, circles, and crosses) in the 3- to 4-mm inner-scale range.

The real experiments were carried out using a $488-\mathrm{nm}$ argon-ion laser with $400-$ to $500-\mathrm{mW}$ power. The propagation length was $1200 \mathrm{~m}$, and the intensity and the inner scale $l_{0}$ of the turbulence were measured simultaneously. These simultaneous measurements allowed the authors to separate the variances of irradiance for different inner-scale ranges. Comparisons between Consortini et al. experimental results and our simulation ones are shown in Fig. 6, Fig. 7 , and Fig. 8. The simulations used a mesh width of $1 \mathrm{~m}$ and $256 \times 256$ points; we considered 3.9-, 7.8-, and $15.7-\mathrm{mm}$ inner scales respectively.

The intensity variances $\sigma_{I}^{2}$ in these figures are presented against the parameter $\beta_{0}$, defined by

$\beta_{0}^{2}=0.496 C_{n}^{2} k^{7 / 6} L_{z t}^{11 / 6}$,

which represents the irradiance variance for a spherical wave in the limit of small fluctuations and in the limit of a Kolmogorov power-law refractive-index spectrum $\left(l_{0}\right.$ $=0)$.

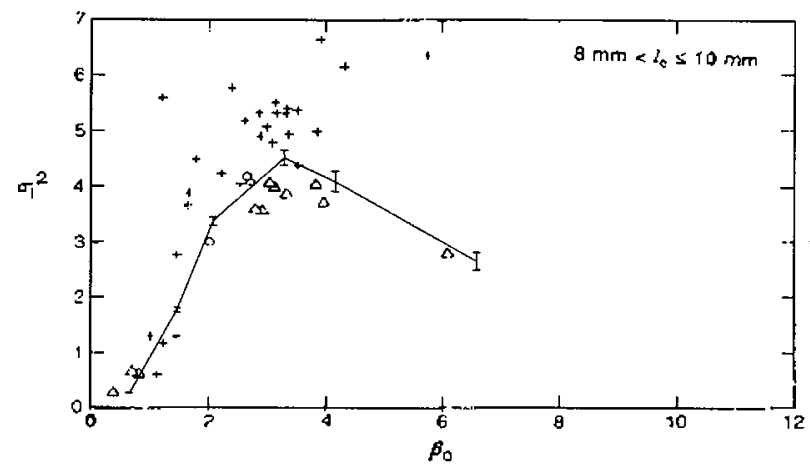

Fig. 7 Variances of the irradiance fluctuations simulated with a 7.8-mm inner scale versus $\beta_{0}$. Simulated results (points connected by lines) are shown superimposed on experimental measurements by Consortini et al. ${ }^{13}$ (triangles, circles, and crosses) in the 8- to 10 -mm inner-scale range.

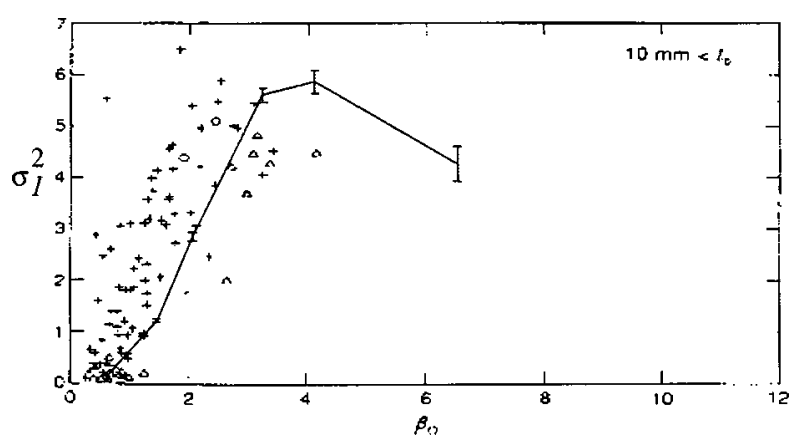

Fig. 8 Variances of the irradiance fluctuations simulated with a $15.7-\mathrm{mm}$ inner scale versus $\beta_{0}$. Simulated results (points connected by lines) are shown superimposed on experimental measurements by Consortini et al. ${ }^{13}$ (triangles, circles, and crosses) in the higher than 10-mm inner-scale range.

Figure 9 shows the saturation of the intensity variances computed for a $17.5-\mathrm{km}$ path, a $512 \times 512$ numerical mesh, and three different inner scales of the turbulence $(2.35,6$, and $9.5 \mathrm{~cm}$ ). The outer scale was fixed at $6 \mathrm{~m}$. The variances of irradiance from the simulations are again presented versus the parameter $\beta_{0}$ defined in Eq. (21). Note that in the saturation regime, the irradiance variances increase monotonically with increasing inner-scale size, as had been found in previous experiments. ${ }^{13}$

The normalized spatial covariance functions obtained from these simulations with an inner scale $l_{0}=6 \mathrm{~cm}$ and different turbulence strengths (Fig. 10) show the typical saturation effect. ${ }^{15}$ As the strength of the turbulence increases, the covariance curves fall off progressively faster, at spacing lesser than $R_{f}=\sqrt{\lambda L}$, and have higher tails than in the weak turbulence case.

Figure 11 presents the theoretical and simulated normalized spatial covariance functions for a $140-\mathrm{km}$ propagation path in a weak turbulence case. We used a 6-m mesh width, $512 \times 512$ points, 100 iterations, six interpolations, and 64 propagation steps. The low structure constant of the index

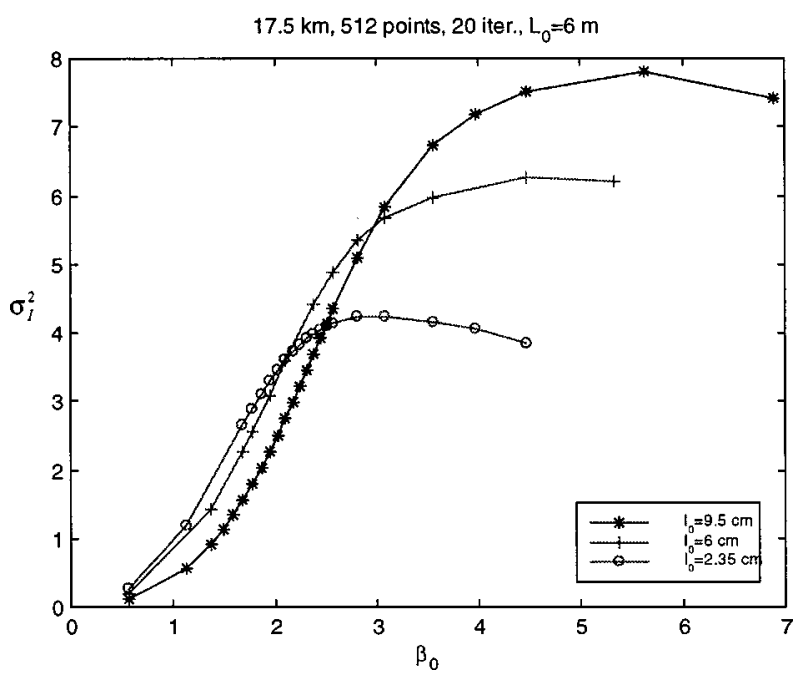

Fig. 9 Simulated strength of scintillation versus the parameter $\beta_{0}$ defined in Eq. (21), for various inner scales $(2.35,6$, and $9.5 \mathrm{~cm})$, path length $17.5 \mathrm{~km}$, and a $512 \times 512$-point mesh. 


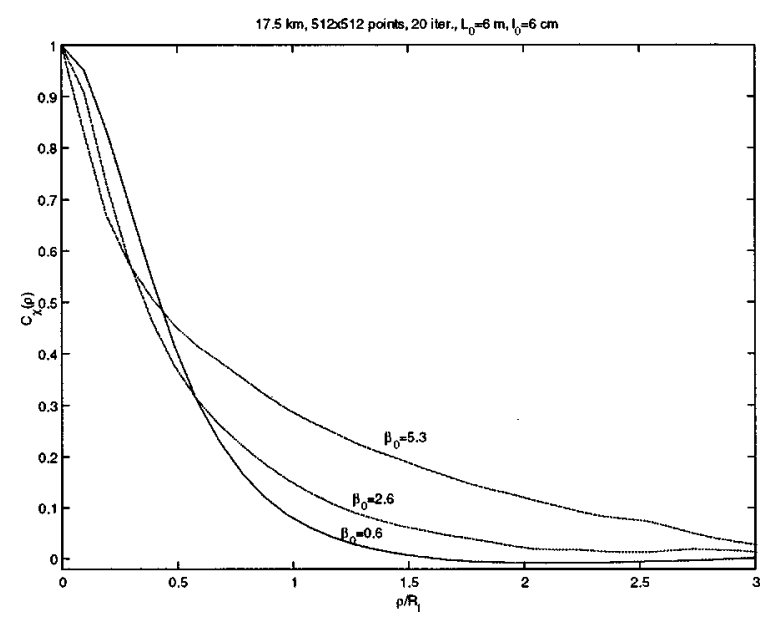

Fig. 10 Three covariance functions of the log-amplitude fluctuations simulated at $17.5 \mathrm{~km}$ under progressively stronger turbulent conditions.

of refraction selected $\left(C_{n}^{2}=10^{-18} \mathrm{~m}^{-2 / 3}\right)$ ensures that the simulation corresponds to a weak turbulence condition, so that it can be compared with theoretical results. The theoretical log-amplitude variance was approximately 3 $\times 10^{-2}$, and its estimated error was less than $10 \%$.

\section{Conclusions}

We have simulated the propagation of spherical waves from a point source through a three-dimensional random medium using a multiple-phase-screen technique. The use of a divergent spherical coordinate system has allowed avoiding the windowing of the beam characteristic of the Cartesian algorithm. To overcome the loss of resolution in the sampled field occurring in long-path propagation simulations, we have proposed a new algorithm that performs repeated interpolations of the field with a zero-padding technique and reduces the angular window to maintain the size of the matrices.

We have compared the simulation results in the weak turbulence regime with estimates based on Rytov's theory. In particular we have calculated errors in the estimated variances of the log-amplitude fluctuations, normalized spatial covariance functions, and coherence diameters.

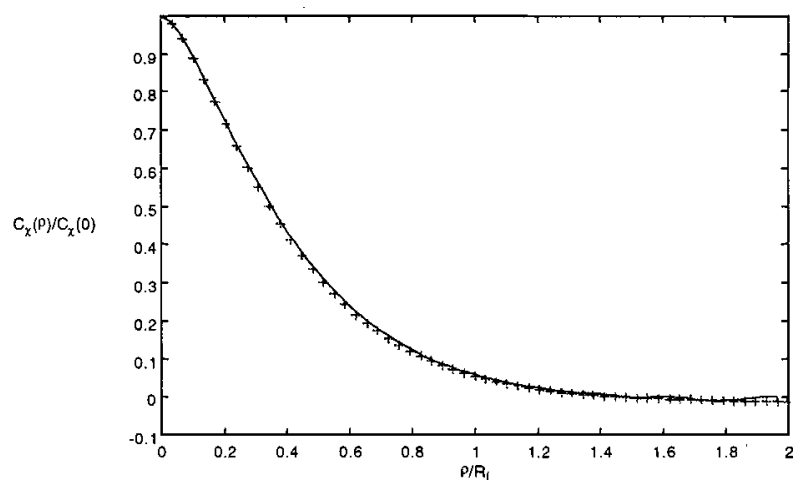

Fig. 11 Normalized spatial covariance functions of log-amplitude fluctuations. Crosses: simulated; continuous line: theoretical. Propagation length $140 \mathrm{~km}, 512 \times 512$ points.
The saturation-range behavior of the simulator has been studied by comparing its results with the experiments carried out by Consortini et al. ${ }^{13}$ We have compared the variances of the irradiance fluctuations for different inner scales of the turbulence. We have also presented curves of the saturation of the log-amplitude variances and normalized spatial covariance functions for long-path propagation simulations.

The comparison of the simulation results with experimental ones and with analytical theory predictions shows very good agreement that confirms the good operation of the proposed algorithm (within the limits imposed by the matrix sizes).

\section{References}

1. V. I. Tatarskii, The Effects of the Turbulent Atmosphere on Wave Propagation, TT-68-50464, U.S. Dept. of Commerce, Springfield, VA (1971)

2. M. E. Gracheva, A. S. Gurvich, S. S. Kashakanov, and V. V. Pokasov, "Similarity relations and their experimental verification for strong intensity fluctuations of laser radiation,' in Laser Beam Propagation in the Atmosphere, J. W. Strohbehn, Ed., Springer-Verlag, New York (1978).

3. J. M. Martin and S. M. Flatté, "Simulation of point-source scintillation through three-dimensional random media," J. Opt. Soc. Am. A 7(5), 838-847 (1990).

4. J. A. Fleck, Jr., J. R. Morris, and M. D. Feit, "Time-dependent propagation of high energy laser beams through the atmosphere," Appl. Phys. 10, 129-160 (1976)

5. W. A. Coles, J. P. Filice, R. G. Frehlich, and M. Yadlowsky, "Simulation of wave propagation in three-dimensional random media,' Appl. Opt. 34(12), 2089-2101 (1995).

6. J. M. Martin and S. M. Flatte, "Intensity images and statistics from numerical simulation of wave propagation in 3-D random media," Appl. Opt. 27(11), 2111-2126 (1988).

7. R. Z. Yahel and I. Last, "Numerical simulation of laser beam propagation in three-dimensional random media: beam splitting and patch formation,' Waves Random Media 2, 81-98 (1992).

8. M. G. Rusbridge, "On the realization of random surfaces," J. Comput. Phys. 2, 288-305 (1968).

9. R. J. Hill and S. F. Clifford, "Modified spectrum of atmospheric temperature fluctuations and its application to optical propagation,' $J$. Opt. Soc. Am. 68, 892-899 (1978).

10. J. W. Strohbehn, "Line-of-sight wave propagation through the turbulent atmosphere,' Proc. IEEE 56(8), 1301-1318 (1968).

11. S. M. Flatté, G. Y. Wang, and J. M. Martin, "Irradiance variance of optical waves through atmospheric turbulence by numerical simulation and comparison with experiment," J. Opt. Soc. Am. A 10(11), 2363-2370 (1993)

12. W. H. Press, S. A. Teukolsky, W. T. Vetterling, and B. P. Flannery, Numerical Recipes in C, The Art of Scientific Computing, 2nd ed. Cambridge University Press, 1992.

13. A. Consortini, F. Cochetti, J. H. Churnside, and R. J. Hill, "Innerscale effect on irradiance variance measured for weak-to-strong atmospheric scintillation,"' J. Opt. Soc. Am. A 10(11), 2354-2362 (1993).

14. W. R. Coles and R. G. Frehlich, "Simultaneous measurements of angular scattering and intensity scintillation in the atmosphere," $J$. Opt. Soc. Am. 72, 1042-1048 (1982).

15. S. F. Clifford, G. R. Ochs, and R. S. Lawrence, "Saturation of optical scintillation by strong turbulence,' J. Opt. Soc. Am. 64(2), 148-154 (1974).

Juan Antonio Rubio received his telecommunication engineer degree from the Telecommunication Engineering School of Barcelona in 1992. From 1992 to 1995 he was a research assistant in the Department of Signal Theory and Communications (TSC) of the Polytechnic University of Catalonia. He is currently an assistant professor in the Electromagnetic \& Photonics Engineering group in the TSC Department and finishing a PhD in engineering. His current research is in free-space optical communications systems and statistical optics.

Aniceto Belmonte received his BS degree in physics from Barcelona University in 1988 and his PhD degree in telecommunications engineering from the Polytechnic University of Catalonia in 1995. His current research interests include statistical optics and all as- 
Rubio, Belmonte, and Comerón: Numerical simulation of long-path spherical wave propagation ...

pects of wave propagation in random media. He has been involved in the design and performance of experiments that showed the suitability of the Canary Islands for hosting an optical ground station for testing and measurement of the intersatellite optical communications payload onboard the European Space Agency's ARTEMIS satellite.

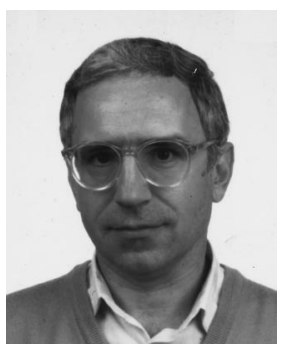

Adolfo Comerón received a telecommunication engineer degree from the Telecommunication Engineer School of Barcelona in 1976, and DEA and Drlng degrees from the Paris-XI University, Orsay, in 1977 and 1980 , respectively. He is currently professor at the Polytechnic University of Catalonia, Barcelona, Spain. His research activities have included the study of nonlinear devices at IR wavelengths and the development of microwave and millimeter-wave receivers for satellite communication systems. They currently focus on free-space optical communications and remote detection and sensing at optical wavelengths. 